\title{
Optimized Routing in Organization-Aware Multihop Wireless Networks (ORION)
}

\author{
Lijun Qian \\ Department of Electrical and Computer Engineering \\ Prairie View A\&M University, Texas A\&M University System \\ Prairie View, Texas 77446, USA \\ Email: liqian@pvamu.edu
}

\author{
Yuanzhu Peter Chen \\ Department of Computer Science \\ Memorial University of Newfoundland \\ St. John's, NL Canada \\ Email: yzchen@mun.ca
}

\begin{abstract}
In our previous works, we defined a novel routing metric for organization-aware multihop wireless networks [1]. We demonstrated that our proposed routing protocol with this new metric provides the much needed survivability and efficiency in battlefield environments when keeping the cross-organization data transfer at a low level [2]. In this paper, we take one step further and propose ORION, a framework for quality-of-service $(\mathrm{Q} O S)$ routing in organization-aware wireless networks. Here, we formulate routing as a constrained optimization problem with a new routing metric to address QoS and security/policy requirements of traffic flows as well as node-specific needs, while taking the organizational constraints into account. Since the problem is not solvable in polynomial time, a near optimal solution procedure based on Lagrangian decomposition and subgradient method is proposed and studied to solve the constrained optimization problem, and such a solution renders itself to distributed implementations. Simulation results show that the proposed method provides high QoS while keeping the crossorganization data transfer at a specified level satisfying strict organizational security constraints.
\end{abstract}

\section{INTRODUCTION}

In many mission critical applications, such as military operations and first responder efforts, multiple organizations are usually involved in the mission. For instance, national guard, firefighters, emergency medical services can be deployed to a disaster region after a severe earthquake. Typically, mobile ad hoc networks (MANETs) are deployed in real time for communications since either the infrastructure may be destroyed or it cannot be accessed. However, different organizations could have heterogeneous wireless networking devices and, yet, information sharing among them plays a pivotal role in saving lives and maximizing operation efficiency. As a result, interoperability among the participating organizations is highly desirable for sharing information and effective collaborations.

The emerging software defined radio (SDR) platform provides an enabling technology for future inter-operable wireless networks used by different organizations [3]. In this paper, we consider the scenario of heterogeneous MANETs deployed within the same geographical area, and assume that all the nodes are inter-operable. Specifically, we focus on the routing problem in scenarios where nodes of one organization can help to transport traffic for nodes of a different organization. This is crucial for maintaining survivability and efficiency in a harsh environment. For example, as illustrated in Fig. 1, the soldiers and the sensors belonging to two different organizations but their radios are inter-operable. Assume that soldier $D$ is out of the radio range of its own network. $D$ could only communicate with other soldiers through sensor node 1 . Moreover, in many cases, more efficient communication may be achieved through collaborations among different organizations. Again, as in the figure, if sensor node 2 has little remaining battery power, we must route the sensed data via other nodes. Without crossorganization data forwarding, we must take the route of 3-45-Fusion Center. However, if the help of the soldiers can be used, it would be more efficient to transport through a shortcut via soldier $A$.

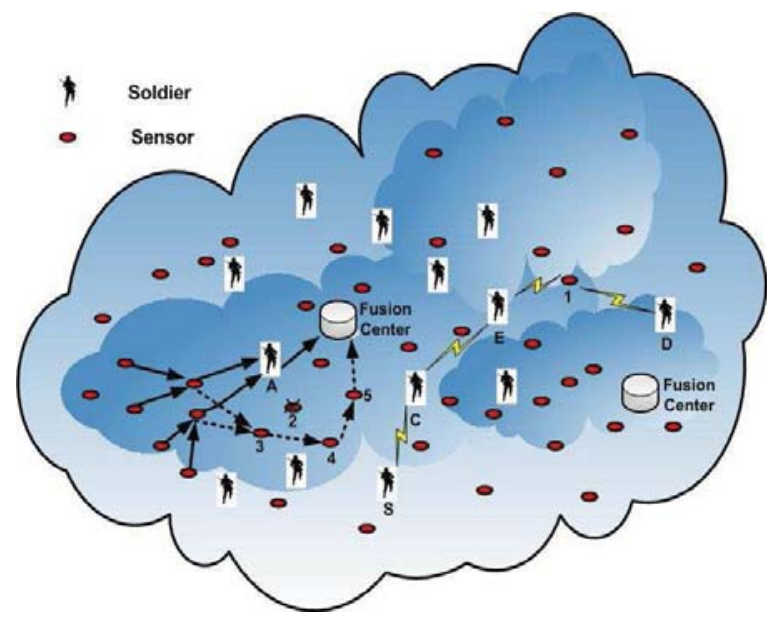

Fig. 1. Collaboration in an organization-aware mobile ad hoc network

Although there can be considerable benefits for different organizations to collaborate in data transfer, it is crucial that the incurred additional overhead to the helping organization will not degrade its own operation significantly. More importantly, the nodes within or outside the same organization may have different security or policy requirements. For instance, suppose that soldier $A$ in Fig. 1 has strict security requirement. In this case, it may not be appropriate for him to relay traffic for the sensor nodes.

In our previous works, we defined an organization-aware routing metric for multihop wireless networks [1]. We demonstrated that such a metric indeed provides the much needed 
survivability and efficiency for mission critical applications while keeping the cross-organization data transfer at a low level [2]. In this paper, we extend our previous results to address Quality-of-Service (QoS) and security/policy requirements of traffic flows as well as node-specific needs, while taking the organizational constraints into account. The proposed formulation strikes the balance between the benefit from collaboration and the cost due to the organization induced constraints. Here, the benefit can be measured by path quality, such as number of hops, data rate or delay. Specifically, we formulate QoS routing as a constrained optimization problem with a new QoS routing metric. Since the problem is not solvable in polynomial time, efficient approximation algorithms are proposed and studied based on Lagrangian decomposition and sub-gradient method. We investigate the effects of the parameter settings on the system performance using extensive simulations using MATLAB. The results demonstrate the effectiveness of the proposed scheme and its capability of addressing the tradeoff between organizational constraints and QoS requirements.

The rest of this paper is organized as follows. The proposed QoS routing metric and the constrained optimization, as well as efficient solutions are described in Section II. Section III is dedicated to simulation studies and our findings out of these experiments. Related works are discussed in Section IV. Section V concludes this article with some discussion on future extensions.

\section{Constrained Optimal QoS Routing}

The mixture of multiple organizations, along with the subgroups therein, can be regarded as one hierarchical network, where it contains a number of tiers of groups. For many mission-critical applications, it is important to keep crossorganization data transfer at the lowest possible level for minimal disruption of the traffic within each organization and for security reasons ("level constraint"). To measure the degree that a multi-hop path conforms to this "level constraint", a new link metric was proposed in [1] to quantify the "distance" between two neighboring nodes in terms of their hierarchical addresses. Then the routing decision in such networks should take into account the "level constraint" such that crossorganization traffic flow will remain at the lowest possible level.

To address the tradeoff between QoS such as end-to-end delay and organizational concerns, we propose a generic routing metric as a weighted sum of both terms. Furthermore, to include hard constraints due to QoS requirements and security and policy issues, we formulate an optimal routing problem as a constrained optimization problem.

\section{A. Generic QoS Routing Metric}

Define a weight vector $\rho=\left\langle\rho_{1}, \rho_{2}, \ldots, \rho_{h}\right\rangle$ that representing how important each level is (due to chain of command, etc.). In addition, a cost that reflecting link quality such as delay or resource consumptions such as power, is denoted by $d_{e}$ and the path cost will be $d_{P}=\sum_{e \in P} d_{e}$. The total cost (including the organization-aware cost and the link quality and resource cost) of a path $P$ can be defined by

$$
C_{P}=\eta_{1}\left[\rho \cdot W_{P}\right]+\eta_{2} d_{P}
$$

where $W_{P}$ is the length of the path (in the organizational sense) [1], $\rho \cdot W_{P}$ denotes the inner product of the two vectors, and $0<\eta_{1}<1,0<\eta_{2}<1, \eta_{1}+\eta_{2}=1$. The goal of the proposed optimal organization-aware routing is (P1)

$$
\min _{P} C_{P}
$$

In other words, for any given source destination pair, find the path such that the total cost of the path is minimized.

Note that the parameters $\eta_{1}$ and $\eta_{2}$ determine the tradeoff between emphasis on organization level constraints and path quality and resource cost. The vector $\rho$ provides further flexibility of specifying the relative importance among each levels.

Problem (P1) is an unconstrained optimization problem. In many practical situations, certain QoS constraints such as endto-end delay bound may be necessary. In addition, there may exist strict organizational security constraints, such as traffic beyond a certain level is not allowed. Hence, we define the following constrained optimization problem: (P2)

$$
\begin{aligned}
\min _{P}\left[\eta_{1} \sum_{i=1}^{h} \rho_{i}\left(\sum_{(j, k) \in P} W_{i}^{(j, k)}\right)+\eta_{2} \sum_{(j, k) \in P} d^{(j, k)}\right] \\
\text { such that } \sum_{(j, k) \in P} d^{(j, k)} \leq d^{P, \text { tar }} \\
\sum_{(j, k) \in P} W_{i}^{(j, k)} \leq W_{i}^{P, \operatorname{tar}} \forall i=1,2, \cdots, h .
\end{aligned}
$$

where $d^{P, t a r}$ and $W_{i}^{P, t a r}$ are upper bounds for QoS (say, delay) and organization security, respectively. In other words, equation (4) and equation (5) reflect the hard QoS requirement and the hard organizational requirement, respectively. The above constraints $\sum_{(j, k) \in P} W_{i}^{(j, k)} \leq W_{i}^{P, t a r} \forall i=$ $1,2, \cdots, h$. states the fact that there is possibly a constraint at each level. If there is no constraint at some level $m$, we may simply set $W_{m}^{P, t a r}=\infty$. It worth noting that since $d_{P}$ represents link quality such as delay and resource consumptions such as power, it is a function of traffic flow [4]. The above (P2) can be translated into the following network flow problem with integer constraints and side constraints:

(P3)

$\min _{(j, k) \in E}\left[\eta_{1} \sum_{i=1}^{h} \rho_{i}\left(\sum_{(j, k) \in E} W_{i}^{(j, k)} x^{(j, k)}\right)+\eta_{2} \sum_{(j, k) \in E} d^{(j, k)} x^{(j, k)}(\phi)\right.$

such that

$\sum_{\{k \mid(j, k) \in E\}} x^{(j, k)}-\sum_{\{k \mid(k, j) \in E\}} x^{(k, j)}= \begin{cases}1 & \text { if } j=s \\ -1 & \text { if } j=t \\ 0 & \text { otherwise }\end{cases}$

$0 \leq x^{(j, k)} \leq 1, \quad \forall(j, k) \in E$

$\sum_{(j, k) \in E} d^{(j, k)} x^{(j, k)} \leq d^{P, t a r}$

$\sum_{(j, k) \in E} W_{i}^{(j, k)} x^{(j, k)} \leq W_{i}^{P, \operatorname{tar}} \forall i=1,2, \cdots, h$.

where $(j, k)$ is the link from node $j$ to node $k$ and $E$ is the set of all links. 


\section{B. Feasibility Conditions and Special Cases}

It is observed that the bounds $\left(d^{P, t a r}\right.$ and $\left.W_{i}^{P, t a r}\right)$ have to be set properly such that problem (P3) would be feasible. Let $W_{i}^{O S P}, \forall i=1,2, \cdots, h$. denote the minimum possible $W_{i}^{P}$ for any path $P$, i.e., it corresponds to the Organizational Shortest Path (OSP). Similarly, let $d^{T S P}$ denote the minimum possible $d^{P}$ for any path $P$. In other words, it corresponds to the Topological Shortest Path (TSP). Here, we use the term "Topological" loosely since it depends on the choice of $d^{(j, k)}$. If it is the same for each link in the network, then indeed $d^{T S P}$ corresponds to the TSP (path with minimum hop count). It is clear that the necessary conditions for a feasible path to exist include:

1) $W_{i}^{P, t a r} \geq W_{i}^{O S P}, \forall i=1,2, \cdots, h$.

2) $d^{P, t a r} \geq d^{T S^{i}}$.

However, unless the OSP and the TSP are identical ${ }^{1}$, the above conditions are not sufficient. Let set $\mathcal{P}^{\text {feasible }}=\{P$ : $W_{i}^{O S P} \leq W_{i}^{P} \leq W_{i}^{P, t a r} \forall i=1,2, \cdots, h ; d^{T S P} \leq$ $\left.d^{P} \leq d^{\bar{P}, \text { tar }}\right\}$. A feasible path for problem (P3) exists iff $\mathcal{P}^{\text {feasible }} \neq \emptyset$. Illustrative examples are given in Section II-C.

If the problem (P3) is feasible, the parameters come into play together with the constraints. Note that this generic path metric degenerates to a few forms depending on the values of $\eta_{1}, \eta_{2}, \rho$, and $d$. These degenerated cases models a number of differing application requirements. Table I summarizes these cases.

TABLE I

DEGENERATION BY DIFFERENT PARAMETER SETTINGS

\begin{tabular}{c|c|c|c}
\hline & Pure OSP & Hop Count & Informative \\
\hline \hline$\eta_{1}$ & any positive number & 0 & 0 \\
\hline$\eta_{2}$ & 0 & any positive number & 1 \\
\hline$d$ & any & same for all links & ETX, ETT, etc. \\
\hline$\rho$ & heavy-head $\rho_{i}$ & any & any \\
\hline
\end{tabular}

It is also interesting to notice that there are multiple parameter settings as well as changing the target value of the constraints may result in the same (optimal) path. For example, there are at least two ways to obtain the TSP assuming that the problem (P3) is feasible: 1) $\eta_{1}=0 ; \eta_{2}$ can be any positive number; and $d$ is the same for all links. 2) $d^{P, t a r}=d^{T S P}$.

\section{Illustrative Examples}

Two small scale examples are presented in this section to illustrate the proposed routing metric and the optimal path found under different constraints and parameter settings. First we use the previous example given in Fig.2 of [2] with the weight vector $W$ indicated on each link. Suppose node $\mathrm{A}$ is the source and node $\mathrm{C}$ is the destination, then $W_{1}^{O S P}=0, W_{2}^{O S P}=4$, and $d^{T S P}=2$ assuming $d=1$ for all the links. Following the discussions in the previous section, the necessary conditions for a feasible path to exist include 1) $W_{1}^{P, \text { tar }} \geq 0, W_{2}^{P, \text { tar }} \geq 4$; 2) $d^{P, \text { tar }} \geq 2$. It is obvious that

\footnotetext{
${ }^{1}$ In this case, the OSP or TSP is the optimal solution to problem (P3).
}

these two conditions are not sufficient. In fact, the solution to problem (P3) does not exist when all the above constraints are tight. In other words, a path with $W^{P}=\langle 0,4\rangle$ and $d^{P}=2$ does not exist. Now, if we loosen one of the constraints, say $d^{P, t a r}=4$, then A-D-E-F-C would be the optimal path and it is the OSP from A to C. If we keep $d^{P, t a r}=2$, and set $W^{P, t a r}=\langle 2,0\rangle$, A-B-C is the optimal path, and it is the TSP from A to $\mathrm{C}$. These are the two extreme cases corresponding to the strict requirements on organizational constraint and QoS (hop count) constraint, respectively.

In general, if the constraints are not binding, the constrained optimization problem (P3) reduces to an unconstrained optimization problem and the optimal path could be different from the OSP and the TSP. An example is given in Fig.2, where node $\mathrm{A}$ is the source and node $\mathrm{D}$ is the destination and the weight vector $W$ and $d$ are marked on each link. Other parameters are set as $\eta_{1}=\eta_{2}=0.5, \rho=\langle 4,1\rangle$. The resulted OSP, TSP and optimal path are given in Table.II. It is clear that in this example, all of them are different.

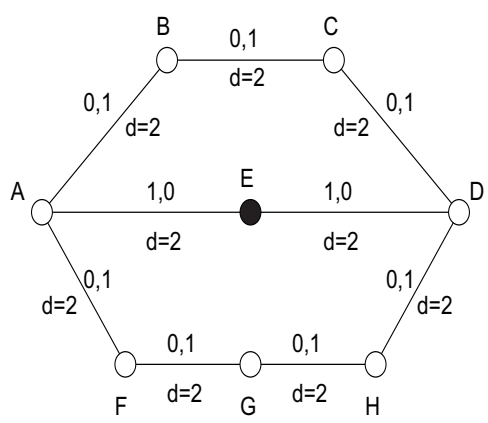

Fig. 2. An example where OSP, TSP and optimal path are all different.

TABLE II

AN EXAMPLE

\begin{tabular}{c|c|c|c}
\hline path & $W^{P}$ & $D^{P}$ & Objective $C^{P}$ \\
\hline \hline OSP: A-B-C-D & $\langle 0,3\rangle$ & 6 & 4.5 \\
\hline TSP: A-E-D & $\langle 2,0\rangle$ & 2 & 5 \\
\hline optimal path: A-F-G-H-D & $\langle 0,4\rangle$ & 4 & 4 \\
\hline
\end{tabular}

\section{Optimal Solution}

We solve the problem (P3) using the dual decomposition technique [5]. The problem (P3) may be rewritten as

$$
\min \sum_{(j, k) \in E}\left(\eta_{1} \cdot \rho \cdot W^{(j, k) T}+\eta_{2} \cdot d^{(j, k)}\right) \cdot x^{(j, k)}
$$

$$
\begin{aligned}
\text { subject to: } & \sum_{k \mid(j, k) \in E} x^{(j, k)}-\sum_{k \mid(j, k) \in E} x^{(k, j)}=\left\{\begin{aligned}
1 & \text { if } j=s \\
-1 & \text { if } j=t \\
0 & \text { otherwise }
\end{aligned}\right. \\
& \sum_{(j, k) \in E} d^{(j, k)} \cdot x^{(j, k)} \leq d^{\text {tar }} \\
& \sum_{(j, k) \in E} \rho^{(j, k) T} \cdot x^{(j, k)} \leq W^{\text {tar }}
\end{aligned}
$$


where $W^{(j, k) T}=\left[W_{1}^{(j, k)}, W_{2}^{(j, k)}, \cdots, W_{h}^{(j, k)}\right]^{T}$. We introduce Lagrange multiplier $\mu$ for each of the side constraints, and we form the corresponding Lagrangian function

$$
L(x, \mu)=f(x)+\sum_{t=1}^{2} \mu_{t} \cdot g_{t}(x)
$$

where $f(x)=\sum_{(j, k) \in E}\left(\eta_{1} \cdot \rho \cdot W^{(j, k) T}+\eta_{2} \cdot d^{(j, k)}\right) \cdot x^{(j, k)}$

$$
\begin{aligned}
& g_{1}(x)=\sum_{(j, k) \in E} d^{(j, k)} \cdot x^{(j, k)}-d^{t a r} \\
& g_{2}(x)=\sum_{(j, k) \in E} \rho^{(j, k) T} \cdot x^{(j, k)}-W^{t a r}
\end{aligned}
$$

Let $\tilde{F}$ denote the conservation flow constraints

$\tilde{F}=\left\{x \mid x \in[0,1], \sum_{k \mid(j, k) \in E} x^{(j, k)}-\sum_{k \mid(j, k) \in E} x^{(k, j)}=s_{j}\right\}$

The dual function is defined as

$$
q(\mu)=\inf _{x \in \tilde{F}} L(x, \mu)
$$

Thus the dual problem can be formulated as

$$
\begin{array}{r}
\operatorname{maximize} q(\mu) \\
\text { subject to } \mu \geq 0
\end{array}
$$

1) Subgradient Algorithm: In this section, a subgradient algorithm is presented to resolve the dual problem (12). The subgradient of $q(\mu)$ at a given $\mu \geq 0$ is defined to be any vector $g$ such that

$$
q(v) \leq q(\mu)+(v-\mu)^{\prime} g, \forall v \geq 0
$$

For any $\mu$, let $x_{\mu}$ to be the minimizer of the Lagrangian function $L(x, \mu)$ over $x \in \tilde{F}$

$$
x_{\mu}=\arg \min _{x \in \tilde{F}} L(x, \mu)
$$

It can be shown that vector $g=\left[g_{1}\left(x_{\mu}\right), g_{2}\left(x_{\mu}\right)\right]$ that has components

$$
\begin{aligned}
& g_{1}(x)=\sum_{(j, k) \in E} d^{(j, k)} \cdot x_{\mu}-d^{\text {tar }} \\
& g_{2}(x)=\sum_{(j, k) \in E} \rho^{(j, k) T} \cdot x_{\mu}-W^{t a r}
\end{aligned}
$$

is a subgradient of $q$ at $\mu$.

Proof: From the definition of $L(x, \mu), q(\mu)$ and $x_{\mu}$, we can get

$$
\begin{aligned}
q(v)= & \inf _{x \in \tilde{F}} L(x, \mu) \leq L\left(x_{\mu}, v\right) \\
= & f\left(x_{\mu}\right)+v_{1} \cdot g_{1}(x)+v_{2} \cdot g_{2}(x) \\
= & f\left(x_{\mu}\right)+\mu_{1} \cdot g_{1}\left(x_{\mu}\right)+\mu_{2} \cdot g_{2}\left(x_{\mu}\right) \\
& +\left(v_{1}-\mu_{1}\right) \cdot g_{1}\left(x_{\mu}\right)+\left(v_{2}-\mu_{2}\right) \cdot g_{2}\left(x_{\mu}\right) \\
= & q(\mu)+(v-\mu)^{\prime} \cdot g
\end{aligned}
$$

Thus, the evaluation of $q(\mu)$ which requires finding a minimizer $x_{\mu}$ of $L(x, \mu)$ over $\tilde{F}$, yields a by-product, the subgradient $g$. The subgradient method consists of the iteration

$$
\mu^{k+1}=\left[\mu^{k}+s^{k} \cdot g^{k}\right]^{+}
$$

where $g^{k}$ is the subgradient of $q$ at $\mu^{k}$ as shown in (15), and $s^{k}$ is a positive scalar stepsize. In this work, we adopt the following stepsize formula

$$
s^{k}=\frac{a^{k}\left(q^{k}-q\left(\mu^{k}\right)\right)}{\left\|g^{k}\right\|^{2}}
$$

where $q^{k}$ is an approximation to the optimal dual cost and $a^{k}$ is a constant within the interval

$$
0 \leq a^{k} \leq 2
$$

Since $q^{k}$ is difficult to estimate, we use the best known upper bound of $q(\mu)$ to the optimal dual cost at the $k$ th iteration, i.e. $q^{k}=\max \left\{q^{1}(\mu), q^{2}(\mu), \cdots, q^{k}(\mu)\right\} . a^{k}$ is a number which is initially equal to one and it decreased by a certain factor for every iteration. The formula for $a^{k}$ is given as

$$
a^{k}=\frac{m}{m+k}
$$

In each iteration, $L(x, \mu)$ can be re-organized as

$$
\begin{aligned}
L(x, \mu)= & \sum_{(j, k) \in E}\left[\left(\eta_{1}+\mu_{2}^{k}\right) \rho \cdot W^{(j, k) T}+\left(\eta_{2}+\mu_{1}^{k}\right) d^{(j, k)}\right] x^{(j, k)} \\
& -\left(\mu_{1}^{k} d^{t a r}+\mu_{2}^{k} W^{t a r}\right), \quad \forall x^{(j, k)} \in \tilde{F}
\end{aligned}
$$

where $\mu_{1}^{k}$ and $\mu_{2}^{k}$ can be regarded as constants. Therefore, $\min _{x \in \tilde{F}} L(x, \mu)$ is reduced to the shortest path problem with non-negative arc lengths. Single origin/destination label correcting algorithm is adopted in this work to find the minimizer $x_{\mu}$ of the $L(x, \mu)$, see Table III. The label correcting method uses a queue to store the candidate list V. Small Label First (SLF) approach is used for node entrance and Large Label Last (LLL) is used as node removal method from queue. To reduce the number of node entrance to the candidate list, we define $u_{i}$ as an underestimate of the shortest distance from node $i$ to node $t$ (destination) for each node $i \in \mathcal{N}$. Since the arc length in this work is non-negative, we let $u_{i}=0, \forall i \in \mathcal{N}$.

2) Convergence and Optimality: We refer to the optimal values attained in the primal and in the dual problems as the optimal primal cost and optimal dual cost, respectively. It is noted that the optimal dual cost is always no greater than the optimal primal cost which is referred as weak duality theorem. It can be given as

$$
\sup _{\mu \geq 0} q(\mu) \leq \inf _{x \in \tilde{F}, g_{t}(x) \leq 0, t=1, \cdots, r} f(x)
$$

There are many results on the convergence of the subgradient method. For constant step size and constant step length, the subgradient algorithm is guaranteed to converge to within some range of the optimal value, i.e., we have

$$
\lim _{k \rightarrow \infty} q_{b e s t}^{k}(\mu)-q^{*}(\mu)<\epsilon
$$


TABLE III

LABEL CORRECTING ALGORITHM

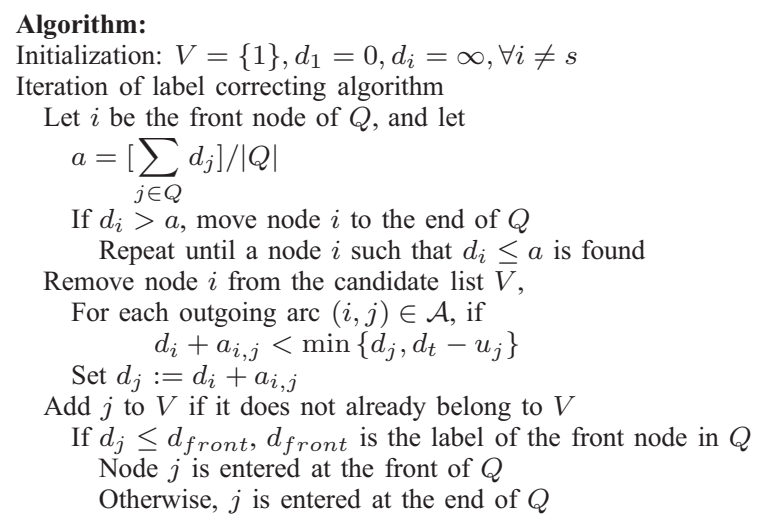

where $q^{*}(\mu)$ denotes the optimal value of the dual problem, i.e., $q^{*}(\mu)=\sup _{\mu} q(\mu)$ and $q_{\text {best }}^{k}(\mu)=$ $\max \left\{q^{1}(\mu), q^{2}(\mu), \cdots, q^{k}(\mu)\right\}$. This implies that the subgradient method finds an $\epsilon$-suboptimal point within a finite number of steps.

\section{Simulation Study}

In this simulation study, we test the proposed constrained QoS routing algorithm in a "city" scenario. It is assumed that there are 500 nodes randomly distributed on 2 west-east streets and 4 north-south streets within a $1500 \times 2500 \mathrm{~m}^{2}$ urban area. The node transmission range is $250 \mathrm{~m}$. The setup is shown in Fig. 3 and the exact configuration is given in the file available at http://nsfrise.pvamu.edu/webpage/files/mmc_street_posall_500.txt. The first 3 columns are the index (node ID), x-position and yposition of the nodes, respectively. In order to simulate realworld organizational hierarchy, for instance, the multi-level tactical networks [6], and test the performance of the proposed algorithm in networks with multi-level hierarchical addresses, we assume a 4-level hierarchy, where each node belongs to one of the 3 different organizations, denoted by addr[0] with values 0,1 , or 2 , and there are 5 branches within each organization and 5 groups within each branch. Each node has a 4-level address similar to the common IP address but with different meaning and much less span. For example, a node with address $\operatorname{addr}[0]=1, \operatorname{addr}[1]=0, \operatorname{addr}[2]=4, \operatorname{addr}[3]=0$, means the node belongs to organization 1 , branch 0 , group 4 , and ID 0 . The organization weight of a link can be calculated by comparing the addresses of the two end nodes. For instance, the weight vector between node $3\langle 1,0,2,3\rangle$ and node $4\langle 1,3,4,4\rangle$ is $W_{34}=\langle 0,1,1,1\rangle$. The weight vector between node 0 $\langle 1,0,4,0\rangle$ and node $3\langle 1,0,2,3\rangle$ is $W_{03}=\langle 0,0,1,1\rangle$. It is assumed that $d=1$ for all the links unless stated otherwise.

\section{A. Finding OSP and TSP}

We first perform a proof-of-concept test by finding OSP and TSP using the proposed algorithm. Specifically, we select

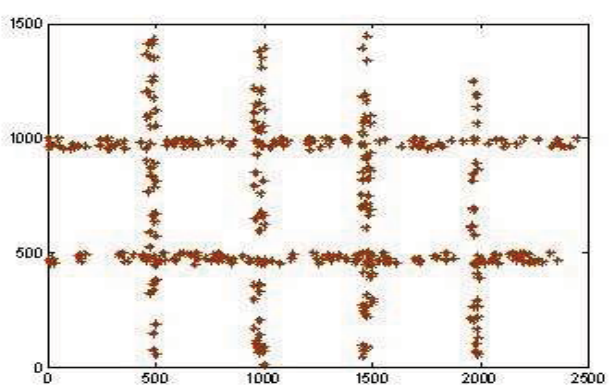

Fig. 3. Simulation setup.

a source and a destination uniformly from 1 to 500 and find the TSP and OSP by solving the unconstrained optimization problem with $\eta_{1}=0, \eta_{2}=1$ for TSP and $\eta_{2}=0$, $\eta_{1}=1, \rho=\langle 64,16,4,1\rangle$ for OSP, respectively. We repeat this experiment 20 times and we record the resulted paths and calculate their corresponding path organizational weight vectors $\left(W^{T S P}, W^{O S P}\right)$ and hop count $\left(d^{T S P}, d^{O S P}\right)$. It is observed that we find the correct TSP and OSP each time, and the average weight (calculated as the product of $\rho$ and $W$ ) is 164 for OSP and 585 for TSP, while the average hop count is 9 for TSP and 12 for OSP. This confirms that the proposed routing algorithm is able to find OSP with significantly less cross-unit traffic while only incur moderate increase in hop count.

\section{B. Effects of Parameters}

As pointed out earlier, the parameters in the proposed QoS routing metric affect the path found by the algorithm. Here we demonstrate the effect of the weight factor $\rho$, by finding the "loose" OSPs with the setting (1) $\eta_{2}=0, \eta_{1}=1$, $\rho=\langle 8,4,2,1\rangle$ for $O S P_{L 1}$ and (2) $\eta_{2}=0, \eta_{1}=1$, $\rho=\langle 4,3,2,1\rangle$ for $O S P_{L 2}$. We record $O S P_{L 1}$ and $O S P_{L 2}$ and calculate their corresponding hop counts. Comparing with the OSP obtained in section III-A by keeping the same sourcedestination pairs, the average hop count of $O S P_{L 1}$ and $O S P_{L 2}$ are 10 and 9 , respectively. It can be seen that with less emphasis on organizational constraints, the hop count of a "loose" OSP can be very close or equal to that of a TSP. In our experiment, the average weight is 35 for $O S P_{L 2}$ and 47 for TSP while they have the same hop count. Hence, a proper setting of parameters may result a path has both low cross-unit traffic and low hop count.

\section{Effects of Constraints}

Here we focus on the effects of the hard QoS and organizational constraints on the resulted paths. We impose very tight constraints: $W_{1}^{P, \text { tar }}=W_{1}^{T S P}, W_{2}^{P, \text { tar }}=W_{3}^{P, \text { tar }}=$ $W_{4}^{P, t a r}=500, d^{P, t a r}=d^{O S P}$, and $\eta_{1}=\eta_{2}=0.5$. Assume that $d$ for each link is a random integer uniformly distributed between 1 and 10, representing random delay (not hop count) of each link. The resulted paths by solving the constrained optimization problem as well as the parameter settings and the 
TABLE IV

CONSTRAINEd Optimal QOS PATHS (HC:HOP COUNT)

\begin{tabular}{l|l|l|l}
\hline$\rho$ & TSP & OSP & obtained path \\
\hline \hline case (1): $\rho=\langle 64,16,4,1\rangle$ & $W=454, d=13, h c=10$ & $W=164, d=57, h c=12$ & converge to OSP (15 iterations) \\
\hline case (2): $\rho=\langle 8,4,2,1\rangle$ & $W=88, d=13, h c=10$ & $W=64, d=18, h c=10$ & converge to OSP (26 iterations) \\
\hline case (3): $\rho=\langle 4,3,2,1\rangle$ & $W=62, d=13, h c=10$ & $W=50, d=18, h c=10$ & converge to OSP (30 iterations) \\
\hline case (4): $\rho=\langle 1,1,1,1\rangle$ & $W=32, d=13, h c=10$ & $W=18, d=48, h c=10$ & nor OSP or TSP (10 iterations) \\
\hline
\end{tabular}

average weights, delay and hop count are listed in Table. IV. It is observed that the proposed algorithm converges to OSP in a relatively large range of parameter settings, cases (1)-(3). In case (1), most emphasis is given to organizational constraints, hence the low weight but high delay of the resulted path. Cases (2) and (3) provide balanced path coincide with OSP, with low weight, low delay and hop count equal to that of the TSP. Case (4) gives a path neither OSP nor TSP with weight and delay in between. From the above experiments, we observe that the proposed method is flexible enough to fit our needs for addressing the tradeoff between QoS requirements and organizational constraints.

\section{RELATED WORK}

Hierarchical routing have been studied extensively for military applications, such as [6], [7]. In [6], an IP multicast routing protocol, called "Hierarchical Level-based IP Multicasting" (HLIM), is proposed for tactical networks. Extended Hierarchical State Routing (EHSR) is proposed in [7] for a multi-level physical heterogeneous network with UAVs. Both papers addressed the routing scalability problem for large networks by arranging the nodes in a hierarchical manner. However, each lower-level node can only communicate with a pre-designated higher-level node or nodes at the same level. Contrary to the configuration in [6], [7], our proposed ORION allow collaborations among all nodes in a mesh topology, while maintaining the organizational structure and satisfying QoS requirements and node specific needs.

QoS routing has also been studied in many previous works, such as in past MILCOM [8], [9], [10], [11], just to name a few. Incorporation of flexible and scalable QoS path selection heuristics into a Network Engineering Design Analytic Toolset (NEDAT) is proposed in [8]. In [9], traffic aware QoS routing is studied and it is shown that Heavy-tailedness Aware Routing Protocol (HARP) significantly outperforms the state-of-theart under heavy-tailed workload. Both [10] and [11] concern inter-domain routing, namely, BGP performance with QoS requirements. In addition, it is noted that the dual decomposition technique has been used to develop distributed routing algorithms, e.g., in [12]. However, none of them considered QoS routing with organizational constraints.

\section{CONCLUSions AND Future Work}

In this paper, we consider a novel QoS routing metric to address one of the critical issues in organization-aware wireless networks, the tradeoff between QoS requirement and organizational constraints. Furthermore, we take into account strict QoS requirement such as the end-to-end delay and hard security constraints and formulate a constrained optimization problem. The dual decomposition method is applied to solve this constrained optimization and a sub-gradient search based algorithm is proposed to obtain the near optimal solution efficiently. Simulation results demonstrate the effectiveness of the proposed method.

Note that any routing protocol implementing the proposed QoS routing metric needs large amount of information propagated through the network, thus introducing burden on the control plane. We can create a multi-point relay for propagating information efficiently using the concept of Connected Dominating Set, which can be calculated efficiently using only local information [13]. Detailed scheme will be designed in our future research. In addition, the effects of node mobility on the proposed routing protocol will also be studied.

\section{REFERENCES}

[1] Y. Chen, L. Qian, and D. R. Vaman., "Hierarchical path metric in multihop wireless networks," in Proc. of IWCMC, August 2008.

[2] Y. Chen and L. Qian., "Organization-aware routing in mission critical networks," in Proc. of IEEE MILCOM, October 2009.

[3] R. North, N. Browne, and L. Schiavone, "Joint tactical radio system connecting the gig to the tactical edge," in Proc. of IEEE MILCOM, October 2006.

[4] J. Chang and L. Tassiulas, "Maximum lifetime routing in wireless sensor networks," IEEE/ACM Transactions on Networking, vol. 12, no. 4, pp. 609-619, August 2004.

[5] D. Bertsekas, Network Optimization: Continuous and Discrete Models. Athena Scientific, 1998.

[6] J. Lee and M. Cheng, "Hierarchical level-based ip multicasting for tactical networks," in Proc. of IEEE MILCOM, October 1999.

[7] D. Gu, G. Pei, H. Ly, M. Gerla, and X. Hong, "Hierarchical routing for multi-layer ad-hoc wireless networks with uavs," in Proc. of IEEE MILCOM, October 2000.

[8] L. Kant and et al., "Network design approaches and tradeoffs in qos route selection with diverse objectives and constraints," in Proc. of IEEE MILCOM, October 2008.

[9] J. Li, S. Luo, W. Tang, R. Levy, and K. Park, "Traffic aware qos routing in ad hoc wireless networks," in Proc. of IEEE MILCOM, October 2007.

[10] E. Kubera, J. Sliwa, K. Zubel, and A. Mroczko, "Validation of qos routing in heterogeneous communication network," in Proc. of IEEE MILCOM, October 2005.

[11] L. Benmohamed, B. Doshi, T. DeSimone, and R. Cole, "Inter-domain routing with multi-dimensional qos requirements," in Proc. of IEEE MILCOM, October 2005.

[12] A. Ribeiro, N. D. Sidiropoulos, and G. B. Giannakis, "Optimal distributed stochastic routing algorithms for wireless multihop networks," IEEE Transactions on Wireless Communications, vol. 7, no. 11, pp. 4261-4272, Nov 2008

[13] J. Wu and H. Li, "On calculating connected dominating set for efficient routing in ad hoc wireless networks," in DIAL-M'99, Seattle, 1999, pp. $7-14$. 